\title{
A spatial Analysis of Crime and Neighborhood Characteristics in Detroit Census Block Groups
}

\author{
Esther Akoto Amoako ${ }^{\mathrm{a}, *}$ \\ a Spatially Integrated Social Science, Department of Geography and Planning, The University of Toledo, OH, USA, \\ Esther.Amoako@rockets.utoledo.edu.
}

\begin{abstract}
:
Crime has an inherent geographical quality and when a crime occurs, it happens within a particular space making spatiality essential component in crime studies. To prevent and respond to crimes, it is first essential to identify the factors that trigger crimes and then design policy and strategy based on each factor. This project investigates the spatial dimension of violent crime rates in the city of Detroit for 2019. Crime data were obtained from the City of Detroit Data Portal and demographic data relating to social disorganization theory were obtained from the Census Bureau. In the presence of spatial spill over and spatial dependence, the assumptions of classical statistics are violated, and Ordinary Least Squares estimations are inefficient in explaining spatial dimensions of crime. This paper uses explanatory variables relating to the social disorganization theory of crime and spatial autoregressive models to determine the predictors of violent crime in the City for the period. Using GeoDa 1.18 and ArcGIS Desktop 10.7.1 software package, Spatial Lag Models (SLM) and Spatial Error Models were carried out to determine which model has high performance in identifying predictors of violent crime. SLM outperformed SEM in terms of efficiency with (AIC:5268.52; Breusch-Pagan test: 9.8402; R2: 16\% \& Log Likelihood: -2627.26) > SEM (AIC: 5275.24; Breusch-Pagan test: 9.7601; R2: 15\% \& Log Likelihood: -2630.6194). Strong support is found for the spatial disorganization theory of crime. High percent ethnic heterogeneity (\% black) and high college graduates are the strongest predictors of violent crime in the study area.
\end{abstract}

Keywords: Violent Crime, Social Disorganization, Autoregressive Model, Spatial Analysis

\section{Introduction}

Crime is a complex event that occurs when the law, an offender and a victim intersect in space and time (Andresen, 2006). All forms of crime whether violent, property, white color or nuisance crime all result in many costs that could be monetary (law enforcement, property loss, insurance, correction, and judiciary), and psychological (victimization and safety). While different crime may have different monetary and psychological effects on society, the reduction of crime generally yields positive desirable outcomes on society.

Within the literature in criminology, social disorganization theory has frequently been employed to analyze geographies of crime. The theory of social disorganization focusses on social deprivation as a major cause of crime. This theory shifts attention from the offender to the environmental factors (Chainey \& Ratcliffe, 2005). This study evaluates this theory as the basis for spatial autocorrelation analysis of violent crime rates in the City of Detroit, USA at the block group level.

The City of Detroit is the largest city in the midwestern state of Michigan. Occupying a total land area of 149 square miles, Detroit ranks 24th-most populous city in the United States. The city has population of 670,031 with 78.3 percent Black/ African American and 14.7 percent white according to Census 2019 estimates. Figure 1 Shows the map of Detroit.

In the United States, crime since the 1990s has generally been on the decline, the national crimes peaked in 1991 at
5,856 crimes per 100,000 people and has been declining ever since. Crime rates do fluctuate from year to year and by the type of crime. For example, homicide rate may go up in a particular year whiles other types of crime remain minimal, in such cases the overall percent rise in crime rates may be down. The FBI data shows that violent crime rate fell $49 \%$ between 1993 to 2019, with large decreases in rate of robbery by $-68 \%$, murder/non-negligent manslaughter $-47 \%$ and aggravated assault $-43 \%$. Meanwhile, property crime rate fell $55 \%$ with large decline in burglary $(-69 \%)$, motor vehicle theft $(-64 \%)$, and larceny theft (-49\%). The Bureau of Justice (BJS) statistics also found 74\% decline in violent crime rate between 1993 and 2019 , while property crime rate fell $71 \%$. Despite the downward trend in crime rates, Detroit ranks at the top of the FBI's annual list of America's most dangerous cities in 2019. While the violent crime rate in Detroit fell in 2019 from 2018, it was still the highest among major cities (FBI, 2019; Nissen, Fox Television Station, 2020).

This study distinguishes itself from other existing literature on geography of crime in four ways. First, few studies have analysed Detroit crime data at such disaggregated spatial scale. Second, both crime data and census data correspond to the same year, this allows for causal relationships between crime and demographic variables to be easily assessed. Third spatial econometrics technique, spatial autoregressive models are explicitly employed in the analysis to identify which model has high performance in the presence of spatial dependence, in contrast to most inferential work on the geography of 
crime that favours classical statistical analysis. Finally, violent crime is investigated to assess the different demographic and socioeconomic attributes at the census block group level of Detroit to assess the predictive power of social disorganization theory for violent crime rates in the study area.

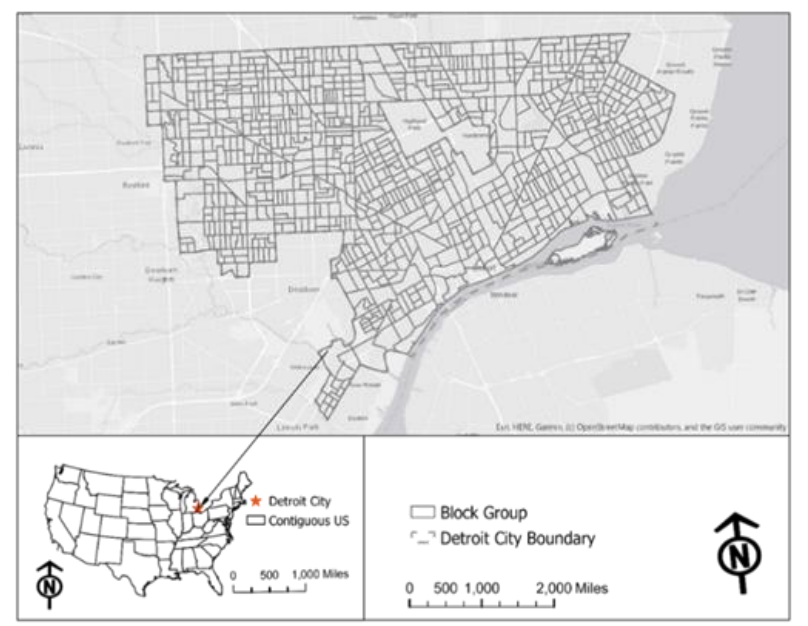

Figure 1. Map of the City of Detroit Census Block Group

\section{Literature Review}

Researchers have long explored the association of crime events with characteristics of the place. Spatial, or ecological or environmental approaches to theory in crime studies can be traced to the work of Guerry and Quetelet in the 19th century. Contemporary work on the spatial dimension of crime is rooted in the work of Shaw and McKay (1942) of the Chicago School. The underlying argument for the theory is that human behavior is situated in place, hence, geography of crime should be a dimension in the analysis. Essentially, this work acknowledges that crime has geographical quality. Within the geography of crime, social disorganization theory is one of the common spatial theories related to the early work.

\subsection{Social Disorganization Theory}

The research that evolved from the work of Shaw and McKay (1942) found strong positive correlation between poverty, social deprivation, and crime. They noted that neighborhood with highest crime rates had at least three common problems including ethnic heterogeneity (high levels of ethnic and cultural mixes), concentrated disadvantage and economic deprivation. Shaw and McKay discovered that delinquent behaviors were direct result of the collapse of community-based controls and people living in disadvantage neighborhoods are responding naturally to their environmental conditions resulting in delinquent behaviors. Several studies have found support for the social disorganization theory (Liu et al., 2016; Varano et al., 2009; Andresen, 2006; Ackerman \& Murray, 2004). The study found strong positive relationship between high unemployment rate and crime. They believed that these factors create disorganization within the community that weakens social control that society exerts on crimes and prevented residents from coming together to solve neighbourhood crime problems leading to the development of delinquents (Shaw \& McKay, 1942). These theories start with the premise that crime can only be understood through the consideration of demographic, economic, and social dimensions of crime, with each factor having their own spatial dynamics.

Several studies have linked crime rates with measurements of poverty, unemployment rates, and neighborhood stability (measured by percentage of rental units, percentage of single parents, etc.). For example, Andresen (2006) found high unemployment rate as the strongest predictor of criminal activity. Feldmeyer, Steffensmeier, and Ulmer (2013) found support for ethnic heterogeneity measure of crime, that racial and ethnic composition of a given area was associated with higher rates of violent crime. Areas were sorted based on the prevalence of African American residents and Latino residents, and findings held consistent for both. Tcherni (2011) found there are structural forces that influence violent crime rates, especially homicide rates, with poverty and low education being the primary factors. One component of the social disorganization theory proposed by Shaw and McKay (1942) is residential stability. Brown \& Weil (2020) found that decreasing marginalization and improving collective efficacy reduced crime rates in neighborhoods.

\section{Crime in Detroit: Data and Methods}

\subsection{Crime Data}

The analytical procedure for this study was conducted using GEODA 1.18 and ArcGIS Desktop 10.7.1.

The data used to measure criminal activities in Detroit are obtained from the City of Detroit Open GIS data Portal. These data reflect reported criminal offenses that have occurred in the City of Detroit. Offense data was extracted from the Detroit Police Department's records management system (RMS data). This data set contains the most recent data available and is updated anytime DPD sends official crime records contributing to the Michigan Incident Crime Reporting (MICR) or the National Incident Based Reporting systems (NIBRS). Accordingly, the data describe all offenses associated with all reported incidents. The RMS crime incidents data covers all reported major crimes within the City from the 1990s to present and it is updated regularly. The attributes include the location, date, time of day, year, offense description, offence category, zip code, Police precincts, neighborhood where the offence occurred as well as the xy coordinate of where the offence was committed.

The main categories of crime that are reported under the Uniform Crime Reporting program (UCR) of the FBI is violent crime and property crime. Violent crimes include rape, non-negligent manslaughter, robbery, and aggravated assault. Property crimes include arson, larceny, burglary, and motor vehicle theft. Only violent crime is considered in this study due to time constraints. The dataset captured 10287 violent crime in Detroit for 2019. There were 276 homicide, 7720 aggravated assault and 
2291 robbery incidence in the period. Violent crime rate was expressed as the total number of violent crimes over the population by 10,000 residents, i.e. (total violent crime/population) $* 10,000$. Counts of violent crime for each block group were generated by means of spatial join in ArcMap. The dependent variable was transformed using square root transformation of violent crime rate as the dependent variable in the model because it assumed a nonlinear relationship between the violent crime and the predictor variables. Also, the decision for square root transformation of violent crime rates was due to skewness within the dataset resulting from many block groups having zero or extremely low crime rates and others having extremely high values of crime. Logarithm of violent crime rates could not be performed due to zero values of crime in many block groups so square root was appropriate for the data transformation.

\subsection{Demographic and Socio-Economic Data}

The socio-demographic and socio-economic data used in this study to represent the social disorganization theory of crime were obtained from the 2019 US Census Bureau ACS (American Community Survey) 5-year estimate. Since the analysis is cross sectional based on 2019 violent crime, the 2019 ACS 5-year estimate census data at the block group level was used to avoid ecological fallacymaking inference using different base years. The variables representing the social disorganization theory are ethnic heterogeneity (measured by percent black/African American population), social/economic deprivation, family disruption, and educational attainment. This study follows previous approaches using the following indicators: ethnic heterogeneity is captured using the percentage of Black/African American population; Social or economic deprivation is measured using unemployment rate, percentage of families receiving food stamps or public assistant, and percentage of households with income below the poverty line; family disruption is measured using the percentage of female headed homes, and the percentage of rental occupied units; educational attainment is measured using the percentage of population with a bachelor's degree or higher, (Xu et al., 2018; Andresen, 2006; Arthur, 1991).

Ethnic heterogeneity (measured by percentage of African American/Black population), unemployment, Female headed families, and rental occupied units have expected positive relationship with violent crime (Andresen, 2006). Education and median household income have expected negative relationship with violent crime rates. It should be noted that, many variables used in this analysis serves as proxies for complex social processes that aids in understanding criminal activity and have proven by empirical study to provide meaningful explanation for crime.

The unit of analysis in this study is at the City of Detroit census block group level. A census block group is the smallest geographical unit for which the bureau publishes sample data. Block groups are statistical divisions of census tracts and are generally defined to contain between
600 and 3,000 people (Census Bureau, 2019). There are officially 879 block groups in the study area, occupying about 142.9 square mile land area. Census block group was selected for spatial extent of the study because it provided a more refine unit to access criminal activities.

\subsection{Model Estimation}

The use of the ordinary least squares (OLS) regression with spatial data is problematic, due to the presence of spatial autocorrelation within the model's residual (Srinivasan, 2008; Anselin, 1989). OLS estimation of spatial data violates the assumption of independent observations. Also, the assumption of uncorrelated error terms is violated. Although the ordinary least squares parameter estimates remain unbiased in the presence of spatial dependence, the estimated parameters remain inconsistent and inefficient (Anselin, 1989). Consequently, spatial econometrics technique has been developed to handle the above situation. Spatial autoregressive model is one of the techniques designed to take into account spatial autocorrelation in estimating parameters to ensure a consistent and efficient estimate.

The study employs exploratory data analysis or data driven approach by deriving information from the data without preconceived idea of theoretical framework. The analysis involved many exploratory data analyses to arrive at a more improved model to determine the predictors of violent crime. Spatial lag and spatial error models were used to determine if the presence of spatial dependence is the result of diffusion process or omitted variable problem. Several diagnostics were performed in GeoDa and ArcGIS Desktop including graphical test (map of squared residuals), heteroskedasticity test was determined by Breusch-Pagan test and diagnostic for spatial dependence was determined by log likelihood ratio test, goodness of fit for the models were determined by log likelihood and Akaike Information Criteria (AIC).

The spatial lag models account for spatial correlation in the dependent variable. The spatial lag models posit that the dependent variable depends on the dependent variable in neighboring spatial units and on a set of observed local characteristics. The general functional form of the spatial lag model is given as:

$\mathrm{y}=\rho \mathrm{Wy}+\mathrm{X} \beta+\varepsilon$,

Where $\varepsilon \sim N\left(0, \delta^{2} I\right)$, with the reduced form:

$\mathrm{y}=(\mathrm{I}-\rho \mathrm{W})-1(\mathrm{X} \beta+\varepsilon)$

where $\mathrm{W}$ is $\mathrm{n} \times \mathrm{n}$ spatial weight matrix (row standardized), $\mathrm{Wy}$, spatially lagged dependent variable, $\mathrm{y}$ is $\mathrm{nx} 1$ dependent variable, $\beta$ is a $\mathrm{k} \times 1$ vector of the model coefficients, $\mathrm{X}$ is an $\mathrm{nx} \mathrm{k}$ matrix of regressors, and $\rho$ is the spatial autoregressive coefficient that determines the strength of spatial autoregressive relationship and $\mathcal{E}$ is identically normally distributed error term with zero mean and variance. With the reduced form, each inverse can be extended into an infinite series, including both the explanatory variables and the error terms at all locations (Anselin, 2003). Therefore, the spatial lag term must be treated as an endogenous variable and proper estimation method must be applied (an OLS estimate will be biased 
and inconsistent due to the simultaneous bias). The spatial lag model is appropriate when the focus is correcting for potentially influence of spatial autocorrelation inherent in spatial data whether the model of interest is spatial or not. The spatial lag model uses maximum likelihood method in model estimation and Queens's contiguity matrix in determining the weight matrix (W).

The spatial error model is a special case of regression with dependence in the error term or spatial dependence is the result of omitted variables. Spatial error model posits that the dependent variable depends on a set of observed local characteristics and that the error terms are correlated across space. The OLS remains unbiased but no longer efficient and the classical estimators for standard error will be biased. It also uses maximum likelihood estimation and queen's contiguity matrix to define neighborhood (W). The general functional form of the spatial error model is given as:

$\mathrm{y}=\mathrm{X} \beta+\mu$, where $\mu=\lambda \mathrm{W} \mu+\varepsilon$

Reduced form: $y=X \beta+(I-\lambda W)-1 \mu$

The same regressors and dependent variables were used in the spatial error model and the parameters are same as before. A general to specific method was employed for final model selection; the model starts with all independent variables (See Table 1) and a square root of violent crime rate was used as the dependent variable. The decision to use square root of violent crime rate instead of violent crime rate was because the variable had unequal variance causing heteroskedasticity in the model.

\section{Results}

\subsection{Descriptive Statistics}

Table 1 shows descriptive statistics of dependent and independent variables used in the spatial autoregressive model estimation. On the average 11.29 violent crime occurred per block group. The maximum number of violent crimes occurring in a block group was 72 . With respect to square root of violent crime, 12.62 crime rates were recorded per block group on the average. Regarding the covariates, on the average there were 48.3 renters occupied units with 40.65 population on food stamps (SNAP) assistance. block groups in the city of Detroit has an average of 82.12 black population and average number of populations with income below poverty line accounted for 6.33 .

Violent crime rate per 10,000 residents is presented in Figure 2. Violent crime rate is concentrated in the Eastern, around the Downtown, and Western part of the city. The Eastern part of the city has historically been occupied by low-income residents and has been associated with high levels of economic and social deprivation which persist. The block groups with high crime values represented in the Eastern Detroit also has high vacant homes with many abandoned houses, and the per capita income is lower than that found in $96.2 \%$ of the neighborhoods in America according to the Neighborhood Scout report, 2021. The clustering of crime around the Downtown area could be attributed to it being the Centre of transportation hub and having many businesses and activities happening there which provide the incentive for offenders and victims to converge in space and time.

\begin{tabular}{lllll}
\hline Variable & Mean & Std. Dev. & Min & Max \\
\hline Total Violent Crime & 11.297 & 7.806 & 0 & 72 \\
Violent Crime Rate & 186.256 & 153.011 & 0 & 1159.42 \\
Square Root of violent crime rate & 12.616 & 5.209 & 0 & 34.050 \\
Percent Rental Units & 48.937 & 21.560 & 0 & 100 \\
Percent SNAP Recipients & 40.654 & 18.363 & 0 & 100 \\
Percent Black Population & 82.124 & 26.098 & 0 & 100 \\
Poverty Rate & 6.332 & 4.651 & 0 & 28.81 \\
Unemployment Rate & 16.693 & 13.5553 & 0 & 78.13 \\
Percent Population with bachelors/higher & 8.550 & 9.556 & 0 & 66.32 \\
\hline
\end{tabular}

Table 1. Descriptive statistics for dependent and independent variables used in spatial regression analysis of violent crime rate in Detroit Census block groups.

Sources: calculations by author

NOTES: Spatial units are Detroit block group ( $\mathrm{n}=879)$, Mapped in figure 1-2

\subsection{Spatial Regression Results}

The results from the spatial autoregressive models are presented in Table 2. Overall, Spatial lag model (SLM) had R-squared value of 16 and spatial error model (SEM) - R squared vale of 15 predicting violent crime rate. The models had 16 percent (SLM) and 15 percent (SEM) respectively, of their variation being explained by the variables representing social disorganization theory. After controlling for spatial dependence, the SLM outperforms the SEM in terms of Akaike Information Criteria (AIC) and Log likelihood. SLM had the lowest AIC of 5270.5 and the highest Log likelihood -2627.25 which makes it the preferred model in estimating predictors of violent crime rate.

The assumption of constant variance of residual is satisfied in both SLM and SEM as indicated by the level of 
significance of the Breusch Pagan diagnostic test of heteroskedasticity results. We fail to reject the null hypothesis assumption of homoscedastic error term; hence, meaningful inference could be made from the study results from models. Inspection of squared residual from the model map is presented in Figure 3. There is statistically significant support to reject the null hypothesis of spatial dependence for all the models after controlling for their spatial relationships using firs-order queen's contiguity to specify the spatial weight matrix, the likelihood ratio test of spatial dependence is statistically significant in the two models. This implies that, the spatial effect of crime and socio-economic factors of social disorganization theory of crime is strong and hence could not be completely removed.

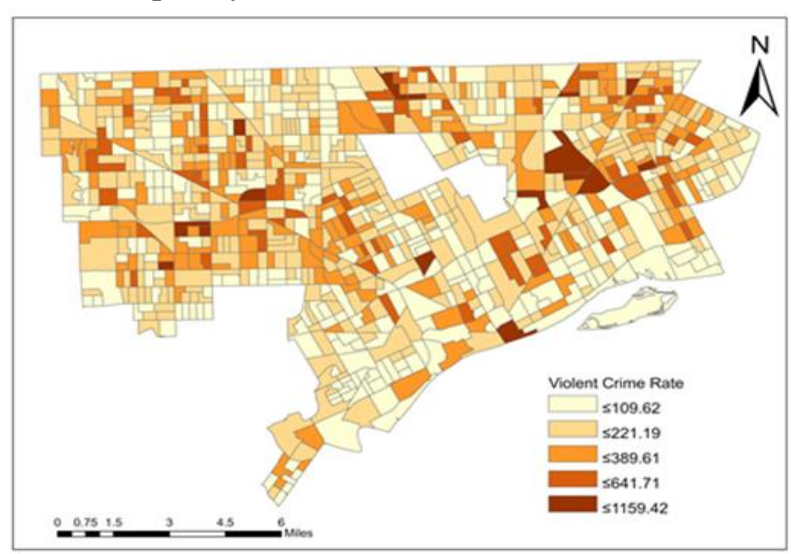

Figure 2. Violent crime rate per 10000, Detroit block group Sources: Map by author

Among all the explanatory variables, percent black population measuring ethnic heterogeneity has statistically significant positive impact on violent crime with about $5 \%$ magnitude of effect. Considering both the magnitude of the estimated coefficients and the ranges of the variables, percent college graduates and percent black population has the greatest impacts and statistically significant effect on violent crime. This finding gives strong support to social disorganization theory of crime that, ethnic heterogeneity has strong positive relationship with crime. The neighborhood crime rate captured by the spatial autoregressive coefficient has strong positive impact on crime both in magnitude and significance, contributing about 22 percent (SLM) and 21 percent (SEM) to neighborhood crime rate.

The percentage of black population has expected positive relationship with violent crime rate, and it is statistically significant. Percentage of college graduates has statistically significant negative correlation with violent crime rate. Both percentage of blacks and college graduates has estimated coefficient greater in magnitude than the other variables and is statistically significant. This implies that, increase in the number of college graduate will lead to about 5 percent reduction in block group unit violent crime rate and when ethnic heterogeneity increases, violent crime rate potentially increases by 5 percent whiles the neighborhood crime rate also increases by 22 percent. Additionally, all the variables in the models retain their correct relationship with violent crime rates, except poverty rate and the percentage of families receiving food stamps assistance. The inference that could be drawn from the result is that in block groups with high ethnic heterogeneity, increase in the number of educated people will lead to a reduction in violent crime rate and vice versa. This is possible as improve education will likely lead to increase employability, improve standard of living and positive social control on criminal behaviors.

\begin{tabular}{ccc}
\hline \multicolumn{3}{c}{ Violent Crime Rate (Square Root of Violent Crime) } \\
\cline { 2 - 3 } Variables & SLM (ML) & SEM (ML) \\
\hline Autoregressive parameter () & $0.225 * * *(0.050)$ & $0.060 * * *(0.008)$ \\
$\%$ Black Population & $0.0499 * * *(0.007)$ & $-0.050^{* *}(0.021)$ \\
$\%$ Bachelors/Higher & $-0.0531 * * *(0.019)$ & $0.007(0.009)$ \\
$\%$ Rental Units & $0.010(0.008)$ & $-0.007(0.040)$ \\
Poverty Rate & $-0.006(0.040)$ & $-0.002(0.011)$ \\
$\%$ SNAP recipients & $0.005(0.011)$ & $0.012(0.013)$ \\
Unemployment Rate & $0.011(0.013)$ & $7.495 * * *(0.833)$ \\
Constant & $5.270 * * *(0.881)$ & $0.207 * * *(0.053)$ \\
LAMBDA & 10.081 & 9.760 \\
Breusch-Pagan & $20.501 * * *$ & $13.760 * * *$ \\
Spatial Dependence (LR) & 0.157 & 0.149 \\
R2 & 5270.5 & 5275.2 \\
AIC & -2627.3 & -2630.6 \\
Log Likelihood & &
\end{tabular}

Table 2. Spatial Regression Result for Violent Crime Rates in Detroit Block Group 
Notes: Spatial units are Detroit block groups $(n=879)$, mapped in Figure $1 \& 2$. All estimated parameters in the spatial regression models have a minimum significance of 5 percent with standard errors in parenthesis. $* * *$ indicates $<0.01$ percent significance and $*$ indicates $<0.05$ percent significance level.

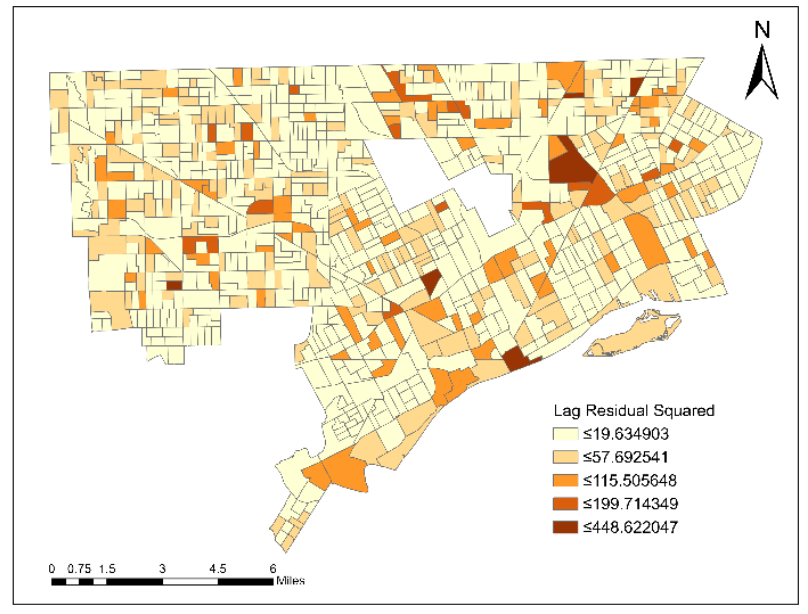

Figure 3. Residual map from the spatial regression of violent crime rate in Detroit block group

\section{Discussions and Conclusion}

The main objective of the study was to assess the effects of socioeconomic factors of the social disorganization theory of criminology on violent crime at the block group level of Detroit. The study employed both the spatial lag and spatial error models to determine the most effective and efficient method in estimating violent crime rates. Based on spatial regression results involving 6 explanatory variables, the conclusion is that socioeconomic factors such as ethnic heterogeneity and the percentage of college graduates are significant predictors of violent crime in Detroit with negative and positive effect on violent crime rates, respectively at the block group level. Thus, block groups with high ethnic heterogeneity (measured by percentage of black population) and high college graduates are likely to result in a reduction in violent crime rate. Conversely, neighborhoods with high levels of ethnic heterogeneity with few educated college graduates will potentially see spikes in violent crime rate. This is because, education will increase the individual's chances of accessing opportunities in the form of high paying jobs which will further improve their quality of life in the neighborhood and the educated people will exert social control on gangs' activities, and criminal behaviors which will yield positive outcomes. On the other hand, studies have shown that most American cities have concentrated socioeconomic disadvantage in neighborhoods with high ethnic heterogeneity: high concentration of immigrants' population, blacks and other minority groups tend to have positive relationship with crime. For instance, Sampson et al. (2005) found concentrated disadvantage, inequality, other structural characteristics and social processes to have strong effects on homicide rate in in predominantly black compared with non-black neighborhoods. The other socioeconomic factors such as unemployment rate, population on SNAP assistance, and renters occupied units has positive effect on violent crime although not statistically significant.

The strong positive effect of percent black population representing ethnic heterogeneity and the positive effect of other factors could also be explain in the context of inequalities and deprivation that exist in most African American and minority neighborhoods in the United States. Black neighborhoods and places with high percentage of immigrant population often have limited opportunities in the form of employment to raise their standards of living. Empirical studies on crime have identified concentrated disadvantage/socioeconomic deprivation to have positive relationship with violent crime and other forms of crime. Existing literature on crime based on the social disorganization theory suggest that neighborhoods with high ethic heterogeneity and concentrated disadvantage tend to have high concentration of crime (Xu et al., 2018; Hipp et al., 2017; Wang \& Arnold, 2008).

The study has revealed some of the systemic issues common among minority and vulnerable populations. Structural disadvantage needs to be improved through targeted policies that aim at improving the employability and standard of living in African American, immigrant and other minority communities. Often, when crime rates increase in a particular neighborhoods, the population who have the means move out leaving behind those who cannot afford to move, and the problem becomes cyclical. There is a need for policy interventions such as skill training, education, and empowerment of communities to play active role in crime prevention by reporting offenders to the police. Law enforcement should build confidence and maintain close relations with residents and build confidence with communities to feel safe in reporting offenders. This measure can significantly contribute to crime reduction.

The study has also demonstrated that, in the presence of spatial dependence, ordinary least square estimation will yield inefficient estimates of parameters and hence spatial autoregressive models that uses maximum likelihood estimates provide consistent and efficient results. Comparing spatial lag and spatial error models, the study proof that, spatial lag model has high performance than spatial error model in predicting violent crime rate. In terms of heteroskedasticity, AIC, log likelihood and R squared all proof spatial lag to have high performance in predicting violent crime rates.

While the strength of the study is demonstrated using spatial autoregressive model to account for neighborhood effects on violent crime rate in each block group unit and establishing percent college graduates and ethnic heterogeneity as strong predictors of violent crime. The limitations of the study should be considered in drawing conclusions and inference from the study. First and foremost, only violent crime rate is considered in the study, all other crime types are beyond the scope of this project. 
Again, the study used aggregated data as against individual crime types. The use of aggregated data as well as focus on single type of crime may account for the insignificant explanatory variables in the model. It is possible, all the variables are good predictors of other forms of crime other than violent crime. Again, the study only focuses on geographical quality of crime while leaving out offenders and law enforcement. The model therefore does not account for the behavior of offenders and how law enforcement guardianship influence on crime. Therefore, the study cannot provide holistic inference on violent crime and generalization of results is applicable in the context of social disorganization theory of crime.

Future studies can explore the relationship between violent crime rates and other theories of criminology such as efficacy and routine activity theory of crime. Additionally, there is a need for further studies to assess the relationship between individual crime types and the explanatory variables to assess their predictive power. Comparison of classical linear regression and spatial autoregressive models could be performed to identify limitations and benefits. The approach used in the study could be replicated at different study area to assess the model's performance.

\section{References}

Ackerman, W. V., \& Murray, A. T. (2004). Assessing spatial patterns of crime in Lima, Ohio. Cities, 21(5), 423-437. https://doi.org/10.1016/j.cities.2004.07.008

Andresen, M. A. (2006a). A spatial analysis of crime in Vancouver, British Columbia: A synthesis of social disorganization and routine activity theory. Canadian Geographer, 50, 487-502.

Andresen, M. A. (2006b). A spatial analysis of crime in Vancouver, British Columbia: A synthesis of social disorganization and routine activity theory. The Canadian Geographer / Le Géographe Canadien, 50(4), 487-502. https://doi.org/10.1111/j.1541-

0064.2006.00159.x

Anselin, L. (1989). What is Special About Spatial Data? Alternative Perspectives on Spatial Data Analysis.

Anselin, L. (2003). Spatial Econometrics. In B. H. Baltagi (Ed.), A Companion to Theoretical Econometrics (pp. 310-330). Blackwell Publishing Ltd. https://doi.org/10.1002/9780470996249.ch1 5

Brown, K. J., \& Weil, F. D. (2020). Strangers in the Neighborhood: Violence and Neighborhood Boundaries. Journal of Contemporary Ethnography, 49(1), 86-117. https://doi.org/10.1177/0891241619857150
FBI. (2019). FBI Releases 2018 Crime Statistics. FBI National Press Office.

Feldmeyer, B., Steffensmeier, D., \& Ulmer, J. T. (2013). Racial/Ethnic Composition and Violence: Size-of-Place Variations in Percent Black and Percent Latino Effects on Violence Rates. Sociological Forum, 28(4), 811-841. https://doi.org/10.1111/socf.12058

Hipp, J. R., Wo, J. C., \& Kim, Y.-A. (2017). Studying neighborhood crime across different macro spatial scales: The case of robbery in 4 cities. Social Science Research, 68, 15-29. https://doi.org/10.1016/j.ssresearch.2017.08. 007

John A. Arthur. (1991). Socioeconomic Predictors of Crime in Rural Georgia. Criminal Justice Review, 16(1).

Liu, D., Song, W., \& Xiu, C. (2016). Spatial patterns of violent crimes and neighborhood characteristics in Changchun, China. Australian \& New Zealand Journal of Criminology, 49(1), 53-72. https://doi.org/10.1177/0004865814547133

Sampson, R. J., Morenoff, J. D., \& Raudenbush, S. (2005). Social Anatomy of Racial and Ethnic Disparities in Violence. American Journal of Public Health, 95(2), 224-232. https://doi.org/10.2105/AJPH.2004.037705

Shaw, R. C., \& McKay, D. H. (1942). The Social Disorganization Theory.

Tcherni, M. (2011). Structural Determinants of Homicide: The Big Three. Journal of Quantitative Criminology, 27(4), 475-496. https://doi.org/10.1007/s10940-011-9134-X

Varano, S. P., Schafer, J. A., Cancino, J. M., \& Swatt, M. L. (2009). Constructing crime: Neighborhood characteristics and police recording behavior. Journal of Criminal Justice, $\quad 37(6), \quad$ 553-563. https://doi.org/10.1016/j.jcrimjus.2009.09.0 04

Wang, F., \& Arnold, M. T. (2008). Localized income inequality, concentrated disadvantage and homicide. Applied Geography, 28(4), 259270.

https://doi.org/10.1016/j.apgeog.2008.07.00 4

Xu, Y., Fu, C., Kennedy, E., Jiang, S., \& OwusuAgyemang, S. (2018). The impact of street lights on spatial-temporal patterns of crime in Detroit, Michigan. Cities, 79, 45-52. https://doi.org/10.1016/j.cities.2018.02.021 


\section{Acknowledgement}

The author of this paper is grateful to David Lilly (Ph.D.)

for useful suggestions and comments on an earlier draft. 